\title{
Mean Free Path and Energy Fluctuations in Quantum Chaotic Billiards
}

\author{
E. Louis ${ }^{1}$, E. Cuevas ${ }^{1,2}$, J. A. Vergés ${ }^{3}$ and M. Ortuño, ${ }^{2}$ \\ ${ }^{1}$ Departamento de Física Aplicada, Universidad de Alicante, \\ Apartado 99, E-03080 Alicante, Spain. \\ ${ }^{2}$ Departamento de Física, Universidad de Murcia, E-30071 Murcia, Spain. \\ ${ }^{3}$ Instituto de Ciencia de Materiales de Madrid, \\ Consejo Superior de Investigaciones Científicas, Cantoblanco, E-28049 Madrid, Spain.
}

(August 7, 1996)

\begin{abstract}
The elastic mean free path of carriers in a recently introduced model of quantum chaotic billiards in two and three dimensions is calculated. The model incorporates surface roughness at a microscopic scale by randomly choosing the atomic levels at the surface sites between $-W / 2$ and $W / 2$. Surface roughness yields a mean free path $l$ that decreases as $L / W^{2}$ as $W$ increases, $L$ being the linear system size. But this diminution ceases when the surface layer begins to decouple from the bulk for large enough values of $W$, leaving more or less unperturbed states on the bulk. Consequently, the mean free path shows a minimum of about $L / 2$ for $W$ of the order of the band width. Energy fluctuations reflect the behavior of the mean free path. At small energy scales, strong level correlations manifest themselves by small values of $\Sigma^{2}(E)$ that are close to Random Matrix Theory (RMT) in all cases. At larger energy scales, fluctuations are below the logarithmic behavior of RMT for $l>L$, and above RMT value when $l<L$.
\end{abstract}

PACS number(s): 72.15.Lh, 05.45.+b

\section{INTRODUCTION}

Quantum systems in which the scattering of carriers by bulk impurities or defects is negligible are traditionally considered as ballistic. An interesting example of these systems are the so called billiards: a region of space in which all scattering occurs at the boundary. The ballistic character of these systems is usually taken for granted, even in the case that their classical analogs behave chaoticallyt. This is so despite of the fact that in wave mechanics the localized character of the scattering centers does not guarantee that their effects be also localized. On the other hand, experimental data for the mean free path of microcavities do always correspond to the bulk material from which the cavity was produced. For instance, Chang et al. 2 give a transport mean free path of the bulk material which is ten times larger than the cavity diameter.

In this work, we characterize quantum billiards by the standard magnitude used in transport studies, i.e., by its mean free path. Surprisingly, the calculated elastic mean free path $l$ is smaller than the linear size of the system for physically relevant values of surface roughness. Afterwards, the consequences of such small values of $l$ are studied on measurable magnitudes like the long-range energy correlations within the spectrum. Our calculations are done for a model of quantum chaotic billiard recently proposed by the authors $\mathrm{B}^{\mathrm{a}}$. The model considers a system which is perfect all throughout the bulk, whereas its surface shows roughness at a microscopic scale. Bulk disorder is not considered since, as remarked above, experimental mean free paths of the bulk systems are usually much larger than cavity diameters. An efficient implementation of this idea was developed in our previous work $\mathrm{k}$ : a tight-binding Hamiltonian with a single atomic level per lattice site in which the energies of the atomic orbitals at the surface sites are chosen at random. We proved that, in the thermodynamic limit, this mode shows the accepted signs of quantum chaotic behavior 1 : an energy spectrum characterized by a Wigner-Dyson distribution of nearest-level spacings, and typical scars in the probability amplitude of wavefunctions.

Section II introduces the quantum billiard model. The elastic mean free path is calculated in two complementary ways in Section III. Long-range energy fluctuations, which are known to be a clear hallmark of diffusive or ballistic behavior $\$$ ed, are analyzed in Section IV. The paper ends with a Section that collects our main results.

\section{QUANTUM BILLIARD MODEL}

Our quantum billiard model is defined by the following tight-binding Hamiltonian:

$$
\widehat{H}=\sum_{i \in S} \omega_{i} \hat{c}_{i}^{\dagger} \hat{c}_{i}+\sum_{\langle i j\rangle} t_{i j} \hat{c}_{i}^{\dagger} \hat{c}_{j},
$$

where the operator $\hat{c}_{i}$ destroys an electron on site $i$, and $t_{i j}$ is the hopping integral between sites $i$ and $j$ (the symbol $\langle i j\rangle$ denotes that the sum is restricted to nearest neighbor sites). We take $t_{i j}=t=-1$ and consider square and cubic lattices for $2 \mathrm{D}$ and $3 \mathrm{D}$ systems, respectively. The energy $\omega_{i}$ of a given atomic level at the surface $(S)$ site $i$, is randomly chosen between $-W / 2$ and $W / 2$, whereas other sites have a constant energy equal to zero. Calculations have been carried out for squares of side up to $L=130$ and for cubes of side up to $L=20$, where $L$ is given in units of the lattice constant. The 
Schwarz algorithm for symmetric band matrices11 was used to compute the whole spectrum.

Note that this model, in contrast with standard billiards 5 , has two length scales: the system size $L$ and the lattice constant $a$. Thus, even in the macroscopic limit $(L / a \rightarrow \infty)$ microscopic roughness remains, and is felt by quantum particles, i.e., particles characterized by a wavelength of the order of $a$.

\section{ELASTIC MEAN FREE PATH}

The ratio $l / L, l$ being the mean free path, is the parameter that controls whether a system is diffusive or ballistic and so the type of fluctuations 10 . The standard definition of the elastic mean free path is 12,13 :

$$
l_{\mathbf{k}} \equiv v_{\mathbf{k}} \tau_{\mathbf{k}}=\frac{\hbar v_{\mathbf{k}}}{2\left|\operatorname{Im} \Sigma\left(\mathbf{k}, \mathbf{k} ; \epsilon_{\mathbf{k}}\right)\right|}
$$

where $\tau_{\mathbf{k}}$ is the relaxation time and $v_{\mathbf{k}}$ the velocity of a state with momentum $\mathbf{k}$. We calculate the selfenergy induced by disorder in an ordered state of momentum $\mathbf{k}$ and energy $\epsilon_{\mathbf{k}}$ from Dyson equation:

$$
\widehat{\Sigma}\left(\epsilon_{\mathbf{k}}\right) \equiv\left\langle\widehat{G}\left(\epsilon_{\mathbf{k}}\right)\right\rangle^{-1}-\widehat{G}_{0}^{-1}\left(\epsilon_{\mathbf{k}}\right),
$$

where $\widehat{\Sigma}$ is the selfenergy operator, $\widehat{G}_{0}$ the unperturbed Green function, and $\langle\widehat{G}\rangle$ the perturbed Green function averaged over disorder realizations. Then, in order to calculate the matrix element $\Sigma\left(\mathbf{k}, \mathbf{k} ; \epsilon_{\mathbf{k}}\right)$, we only have to determine the inverse of the averaged perturbed Green function in the $\mathbf{k}$-space basis using its site representation:

$$
\left\langle\mathbf{k}\left|\left\langle\widehat{G}\left(\epsilon_{\mathbf{k}}\right)\right\rangle^{-1}\right| \mathbf{k}\right\rangle=\sum_{i, j} \psi_{\mathbf{k}}\left(\mathbf{r}_{i}\right) \psi_{\mathbf{k}}\left(\mathbf{r}_{j}\right)\left\langle G\left(i, j ; \epsilon_{\mathbf{k}}\right)\right\rangle^{-1},
$$

where $\psi_{\mathbf{k}}\left(\mathbf{r}_{i}\right)$ are the wave functions of the ordered cluster:

$$
\begin{gathered}
\psi_{\mathbf{k}}^{2 D}\left(\mathbf{r}_{i}\right)=\frac{2}{L+1} \sin \left(k_{x} x_{i}\right) \sin \left(k_{y} y_{i}\right), \\
\psi_{\mathbf{k}}^{3 D}\left(\mathbf{r}_{i}\right)=\frac{2 \sqrt{2}}{(L+1)^{3 / 2}} \sin \left(k_{x} x_{i}\right) \sin \left(k_{y} y_{i}\right) \sin \left(k_{z} z_{i}\right) .
\end{gathered}
$$

It should be noted that non-diagonal elements in Eq.(4) are essential in this case, due to the strong breaking of translational invariance introduced by surface disorder. On the other hand, we have checked that a calculation of the selfenergy due to bulk disorder without the incorporatipn gf non-diagonal elements gives very accurate results 12.13.

The qualitative behavior of 1 can be obtained by second-order perturbation theory 2 . Averaged real-space matrix elements of the selfenergy operator are approximately given by:

$$
\Sigma\left(i, j ; \epsilon_{\mathbf{k}}\right) \approx \delta_{i j}\left\langle\omega_{i}^{2}\right\rangle G_{0}\left(i, i ; \epsilon_{\mathbf{k}}\right),
$$

for those sites $i$ for which the diagonal energy $\omega_{i}$ fluctuates, i.e., for the surface sites. Using the basis given by Eq.(5) to transform to $\mathbf{k}$-space and assuming a constant density of states $(1 /(4 d)$, being $d$ the dimension of the physical space) to get a rough approximation for the imaginary part of the diagonal elements of the unperturbed Green function $\left(\operatorname{Im}\left[G_{0}\left(i, i ; \epsilon_{\mathbf{k}}\right)\right] \sim \pi /(4 d)\right)$, we obtain:

$$
\operatorname{Im} \Sigma\left(\mathbf{k}, \mathbf{k} ; \epsilon_{\mathbf{k}}\right) \approx \frac{\pi}{24} \frac{W^{2}}{L} .
$$

Substituting the selfenergy in Eq.(2) for this approximate value and using the $2 \mathrm{D}$ dispersion relation of the band $\left.e_{\mathbf{k}}=-2\left[\cos \left(k_{x}\right)+\cos \left(k_{y}\right)\right]\right)$ to get an explicit equation for $\hbar v_{\mathbf{k}}$, we finally have:

$$
l_{\mathbf{k}} \approx \frac{24}{\pi} \frac{L}{W^{2}} \sqrt{\sin ^{2}\left(k_{x}\right)+\sin ^{2}\left(k_{y}\right)} .
$$

The final expression for $l$ only uses dimensionless magnitudes, i.e., disorder energies $W$ are measured in units of the hopping energy $t$, lengths in units of the lattice constant $a$, and $\mathbf{k}$ in units of $a^{-1}$. The corresponding expression for $3 \mathrm{D}$ has an additional term within the square root.

When second-order perturbation theory is used to get an approximate value of the mean free path indyced by bulk disorder, a rather good approximation 12 . 23 is obtained due to the homogeneous character of both the perturbation and the real-space Green function. Nevertheless, when the same scheme is applied to billiards, it gives the correct $L$ and $W^{-2}$ dependences but fails to describe the variation of $l$ within the band (see our numerical results shown in Fig.11).

Comparison of the mean free path of a billiard (Eq.(8)) with the result obtained for bulk disorder 12 reveals an essential feature of the present model: whereas for bulk disorder $l$ is independent of the system size $L$, in the present case it depends linearly on $L$. This result is a consequence of having the scattering centers located at the surface, an essential feature of billiards. Therefore, ballistic $(l \geq L)$ or even clean behavior $(l \gg L)$ could naively be expected over the whole band for any value of $W$. Our results will show that this is not the case for physically relevant values of $W$.

Fig.1 1 shows typical numerical results for the mean free path in $2 \mathrm{D}$ and $3 \mathrm{D}$. Note that $l$ is indeed proportional to the system size $L$ in agreement with the approximate result reported in Eq.(8). The dependence of the mean free path on the energy reflects the nature of the eigenvalues discussed in our previous works 3 . In $2 \mathrm{D}, l$ increases near the edges and at the center of the band, due to the presence of quasi-ideal states in these energy regions 14 . Quasi-ideal states do also appear in three dimensions near the band edges and this fact is also reflected in the behavior of the mean free path. The energy 
dependence of $l$ is significantly weaker than in the case of bulk disorder. Actually, $l$ is almost constant except near the band edges and the band center.

Eq.(8) shows that the mean free path monotonically decreases with $W$. However, we note that the definition of the mean free path (Eq.(2)) is intrinsically perturbative. As a consequence, the results for large $W$ cannot be trusted. In fact in the limit of large $W$ the surface decouples from the bulk, leaving a fully ordered cluster of linear size $L-2$. Consequently, the mean free path of the inner part should tend to infinity. In order to handle this problem, we have calculated the mean free path in the large $W$ limit by considering the perturbation of the ordered states of the $(L-2)^{d}$ cluster due to the addition of the surface layer.

Firstly, the averaged Green function of the whole cluster of lineal size $L$ is calculated for the eigenenergies of the smaller cluster of linear size $L-2$. Secondly, the averaged Green function of the inner cluster is obtained using a projection operator $\widehat{P}$, and finally, the selfenergy operator is defined by means of an equation quite similar to Eq.(3):

$$
\widehat{\Sigma}\left(\epsilon_{\mathbf{k}}\right) \equiv\left[\widehat{P}^{\dagger}\left\langle\widehat{G}\left(\epsilon_{\mathbf{k}}\right)\right\rangle \widehat{P}\right]^{-1}-\widehat{G}_{0}^{-1}\left(\epsilon_{\mathbf{k}}\right) .
$$

Since Eq.(9) is an operator definition, any basis can be used in the numerical calculation. We use the tightbinding basis in which the projector $\widehat{P}$ is particularly simple. Thus, the diagonal elements of the selfenergy in the $\mathbf{k}$-space basis are given by:

$$
\left\langle\mathbf{k}\left|\widehat{\Sigma}\left(\epsilon_{\mathbf{k}}\right)\right| \mathbf{k}\right\rangle=\sum_{i, j} \psi_{\mathbf{k}}\left(\mathbf{r}_{i}\right) \psi_{\mathbf{k}}\left(\mathbf{r}_{j}\right) \Sigma\left(i, j ; \epsilon_{\mathbf{k}}\right)
$$

This alternative perturbation theory provides us with a second value of the mean free path. Of course, the larger value is the only one which is physically relevant (perturbation theory logically starts from the less perturbed system). Although we have not found an analytic approximation for this second value of $l$, our numerical results show that $l$ is indeed linearly dependent on $L$ (see Fig.2). Apart from some features that can be related to singularities of the density of states, this new value of $l$ behaves in the same way as the standard value: it shows considerable fluctuations around a mean value that is almost constant within the band. Similar results have been found for three dimensional billiards.

Fig. 3 shows the scaling behavior of both values of $l$ with disorder. The relevant (larger) values of $l$ closely follow an $W^{-2}$ law for small $W$ values while the scaling is close to $W$ for large $W$ values. These scaling laws do not depend on the system size. On the contrary, adding the external shell to the cluster is a perturbation that decreases in importance as the cluster increases (see the behavior of triangles in the left part of Fig 3). Our results show that the ratio $l / L$ can reach values as small as 0.4 close to $W=6$. We will see in the next section, that the value of this ratio strongly determines the behavior of long-range correlations within the energy spectrum. Our data show that the scaling behavior given in Fig. 3 for two dimensional billiards holds for billiards in spaces of larger dimension. This result is not surprising according to well-known results for bulk disorder: mean free path is weakly dimension dependent in those regions in which it is almost constant 13 .

Let us mention that although nearest-neighbors spacing statistics has been proved to follow Wigner-Dyson statistics in our previous work 3 , finite clusters only show "nice" GOE statistics for a range of $W$ values close to the bandwidth. Fig. illustrates this fact. It shows the variance of nearest-neighbor spacing distribution as a function of $W$ for several system sizes. As expected, chaotic behavior is not found when disorder is either very strong or very weak. Beyond a value of $W \approx 10$ which is of the order of the band width, the variance becomes larger than the value corresponding to the Wigner-Dyson statistics (0.286), indicating that the surface starts to decouple from the bulk. For this value of $W$, most of the energy levels which are mainly localized on surface sites lie outside the band and follow Poisson statistics. Also for small $W$ values, the value becomes larger than 0.286 . This indicates a clean behavior of our system: statistics of clean (ordered) systems is more or less Poisson up to some energy scale 15 . The dependence of the variance on the system size is also illustrated in Fig. 1 . When $L$ increases from 32 to 90 , the lower value of $W$ below which the variance is no longer that of GOE decreases. Further increases of $L$ would reduce to zero the widths of the energy regions where quasi-ideal states appear, and thus, the variance would become that of GOE through the whole band, no matter how small $W$ would be.

\section{ENERGY FLUCTUATIONS}

In this section we analyze the relationship between mean free path and measurable physical magnitudes. Although conductance was our first candidate, finally we chose long-range energy fluctuations due to the importance they have in analyzing quantum chaotic systems. Moreover, while the behavior of energy fluctuations within the spectrum is an intrinsic property, conductance depends on the way the system is attached to the metallic leads.

The variance of the number of levels in a given energy interval is a well-known statistics usually referred as $\Sigma^{2}(E)$ statistics $\mathrm{E}$. The standard way of measuring $\Sigma^{2}(E)$ proceeds firstly to transform real spectra onto unfolded spectra: each real spectrum $\left\{\epsilon_{i}\right\}$ is mapped onto an unfolded spectrum $\left\{E_{i}\right\}$ through $E_{i}=\bar{N}\left(\epsilon_{i}\right)$, where $\bar{N}(\epsilon)$ is the averaged number of levels up to an energy $\epsilon$. Secondly, the variance of the number of levels found in an interval of fixed length $E$ is directly obtained from the unfolded spectra. In this Section, we present results for the variance of the number of levels obtained by a dif- 
ferent numerical procedure. Of course, we have checked that overall features are procedure independent, i.e., our method coincides with the standard one when subtleties are ignored. Nevertheless, fine details of the number variance are better given by a more direct definition.

Let us sketch the numerical procedure. Some energy interval $\left[\epsilon_{1}, \epsilon_{2}\right]$ of the band spectrum is chosen. The mean number of levels within the interval is given by:

$$
\bar{N}=\left\langle N\left(\epsilon_{2}\right)-N\left(\epsilon_{1}\right)\right\rangle_{W},
$$

where $N(\epsilon)$ gives the total number of states below energy $\epsilon$ for a generic disorder realization and averaging over disorder configurations is done. In the same way, the mean of the squared number of levels is given by:

$$
\overline{N^{2}}=\left\langle\left[N\left(\epsilon_{2}\right)-N\left(\epsilon_{1}\right)\right]^{2}\right\rangle_{W} .
$$

The variance of the number of levels contained in the energy interval $\left[\epsilon_{1}, \epsilon_{2}\right]$ is simply:

$$
\Sigma^{2}(\bar{N})={\overline{N^{2}}}^{2} \bar{N}^{2} .
$$

In this way, just a value of the $\Sigma^{2}(E)$ function is obtained. This sequence is repeated a large number of times for randomly selected energy intervals until a relatively smooth value is obtained for the variance of the number of levels averaged over energy intervals containing the same number of levels. The last step implies averaging the number variance over some selected region of the energy band.

Note that only fluctuations induced by disorder are taken into account by our method. Therefore, number fluctuation is strictly zero for an isolated spectrum. On the other hand, the standard method can be used even in this situation. The variance is defined by:

$$
\Sigma^{2}(E)=\left[N\left(E_{0}+E\right)-N\left(E_{0}\right)-E\right]^{2} .
$$

The subtlety within this definition is the way followed for unfolding just one spectrum. Sometimes, the asymtotic form of $N_{\epsilon \rightarrow \infty}(\epsilon)$ (Weyl formula) $\sqrt{6}$ is used even in the lower part of the spectrum and number fluctuations are measured relative to $N_{\epsilon \rightarrow \infty}(\epsilon)$. Most frequently, a large number of levels is used to measure the average level spacing and then fluctuations relative to the mean value are measured in smaller intervals. In this way, it has been proved that crystalline spectra are uncorrelated to some energy extent, and consequently, $\Sigma^{2}(E)$ statistics is Poisson 15 :

$$
\Sigma_{\text {Poisson }}^{2}(E)=E \text {. }
$$

Let us remark that our procedure is quite appropriate for billiard ensembles as it is our case (surface roughness is random and several samples can be generated for a given value of surface disorder $W$ ). On the other hand, it gives no fluctuations for conventional billiards (just one spectrum).
We have checked that our numerical procedure recovers both the analytical results for random series of energy levels (see Eq.(15)) and for the eigenvalue series of matrices belonging to the Gaussian Orthogonal Ensemble (GOE)16:

$$
\Sigma_{\mathrm{GOE}}^{2}(E) \approx \frac{2}{\pi^{2}}\left[\ln (2 \pi E)+1.5772-\frac{\pi^{2}}{8}\right] .
$$

The logarithmic dependence on $E$ makes this value much smaller than the one corresponding to uncorrelated spectra. The well-known spectral rigidity of GOE follows: the number of levels in any energy interval is very close to its mean value.

As said in the last part of the previous Section, we routinely calculate the variance of the nearest-level spacing distribution in order to check that the Wigner-Dyson value of 0.286 is recovered by our numerical code. This is always the case for "reasonable" values of $W$ (see Fig. A) in spite of dealing with systems of finite size. Notice that the calculation of $\Sigma^{2}(E)$ for very small values of $W$ is difficulted by finite size oscillations in the density of states 17 .

Fig. 5 shows the typical behavior of the number variance $\Sigma^{2}(E)$ in different transport regimes. Results correspond to the spectra of a large collection of $32 \times 32$ clusters (one thousand samples). All states within the central part of the band $(-3 \leq \epsilon \leq 3)$ have been considered. The black thin line corresponding to $W=2$ shows a typical ballistic behavior: fluctuations are really small in a large energy interval (the number of levels fluctuates in less than one level for intervals containing 100 levels). On the other hand, the black thick line shows a typical diffusive result that corresponds to $W=10$. In this case, number fluctuation follows GOE statistics for a small number of energy levels $(\approx 8)$, remains below GOE up to about 35 levels, and is well above GOE values at larger energy scales. In any case, fluctuations are still much smaller than Poisson values $\left(\Sigma_{\text {Poisson }}^{2}(100)=100\right)$. The gray thick line shows results corresponding to an intermediate situation $(W=6)$. A natural physical explanation of such different behaviors is provided by the ratio of the mean free path to the cluster side: $l / L$ is about 0.7 for the diffusive system but 2.6 for the ballistic one. Although both values are not too far from the accepted assumption $l \sim L$, long-range energy correlations within the spectrum are quite different. Our results for the intermediate value of $W$ prove that the transition from one regime to the other is smooth.

Let us further comment our numerical result for bidimensional billiards of side $L=32$ (Fig.5). Firstly, a continuous increase of the number of levels following GOE statistics is observed for increasing values of $W$ $(W=2,6,10)$. Secondly, it follows a region in which $\Sigma^{2}(E)$ deviates from GOE showing a saturation effect. And finally, an almost linear increase of the variance follows at larger energies. Our qualitative interpretation of this behavior is the following. Strict GOE statistics is 
limited by the maximum degeneracy of crystalline bands. For a 2D cluster of the square lattice with open boundary conditions, maximum degeneracy is 2 although splitting of the 8-fold levels corresponding to periodic boundary conditions is really small. Surface roughness produces a mixing of this degenerate states that is well described by the random matrix elements of matrices of the GOE. This suffices to give Wigner-Dyson statistics for the nearestneighbor spacing distribution. When larger energy scales are considered, the fact that crystalline levels range from energy -4 to 4 implies an incomplete mixing of levels (in the limit of very small non-diagonal random coupling, $\Sigma^{2}(E)$ would be constant beyond the number of levels corresponding to maximum degeneracy). This explains saturation with variance values below GOE statistics. The almost linear Poisson-like increase of the number variance at larger energy is a subtle effect associated to the randomness of the energy levels at the surface. Energy levels suffer uncorrelated shifts due to the formation of surface resonances at uncorrelated energies. It is clear that for a large enough value of $W$, about $4 L$ surface states appear outside the band. For the same reason, about $4 L$ surface resonances exist within the band for smaller $W$ values. While saturation can be easily checked using matrices of random non-diagonal elements but fixed diagonal elements, the Poisson-like linear increase found at larger energies is a characteristic feature of our model.

Similar results are obtained in 3D as shown by Fig.6. A large collection (one hundred samples) of clusters with random surface roughness has been fully diagonalized and the number variance has been calculated. Fluctuations have been obtained for the central part of the band excluding the band center: $-4 \leq \epsilon \leq-2$. In this case, ballistic regime is clearly obtained for $W=2$ (black thin line) whereas diffusive behavior is got for $W=10$ (black thick line). An intermediate behavior is obtained for $W=4$ (gray thick line). Results can be interpreted as in 2D. Strict GOE statistics is always followed by at least six levels (typical 3D crsytalline degeneracy for open boundary conditions). A region showing saturation of $\Sigma^{2}(E)$ follows (this is clearer for $W=2$ than in the other two cases). At larger energy scales an uncorrelated behavior of the variance can be seen. The larger slope of the linear region found for $W=10$ as compared to $W=2$, reflects the fact that surface resonances are better defined in the first case. About 125 energy levels closely follow GOE statistics for the intermediate degree of surface roughness $(W=4)$. We believe that this property is related to a minimum value of the $l / L$ ratio. The smallest value of the mean free path at fixed cluster size is obtained when the mixing of crstalline states is maximum. Therefore, a larger number of energy levels shows correlations that are well described by RMT. Generally speaking, ballistic behavior $(l \geq L)$ corresponds to a minimum mixing of energy levels (only degenerate states are mixed by the surface random perturbation) while diffusive behavior $(l \leq L)$ is seen when surface resonances dominate the value of the number variance.

Fig.7 shows the increase in the number of strongly correlated energy levels for a fixed surface roughness $(W=2)$ as the size of the system increases. In this Figure, a clear discontinuous change of slope can be seen for both curves. About 140 and 300 levels are strongly correlated for $L=14$ and 20, respectively, before the effect of uncorrelated surface resonances dominates. One could naively suggest an $L^{2}$ scaling for the number of correlated levels. Nevertheless, a precise assesment about the scaling of such critical energy would require further merical simulations well beyond our actual possibilities 18 .

\section{CONCLUDING REMARKS}

We have found that the mean free path in the model of quantum chaotic billiards discussed in this work can be substantially smaller than the system size. Actually, the ratio $l / L$ reaches a minimum value of about 0.5 for the most relevant disorder values ( $W$ of the order of the band width). The transition from ballistic to diffusive behavior is governed by the disorder parameter $W$ which simulates surface roughness at a microscopic scale. The behavior of the number variance statistics $\left(\Sigma^{2}(E)\right.$ statistics) clearly distinguishes between both regimes. After closely following RMT for a small number of energy levels, $\Sigma^{2}(E)$ shows a tendency to saturation before increasing in an almost linear way at larger energies. The region of saturation extends up to a really large number of levels in the ballistic regime, while the linear increase quickly dominates in the diffusive regime. Therefore, $\Sigma^{2}(E)$ is roughly below or above the values corresponding to GOE statistics for ballistic or diffusive regimes, respectively. In any case, both transport regimes are still characterized by having a mean free path of the order of the linear size of the system and $\Sigma^{2}(E)$ values much smaller than $E$, i.e., much smaller than values corresponding to completely uncorrelated energy spectra. The behavior of the number variance has been related to the structure of the particular type of random matrices produced by surface disorder. Preliminary studies show that these results are not restricted to the particular model analyzed in this work since similar results been found for a model with real surface roughness 19.

\section{ACKNOWLEDGMENTS}

This work was supported in part by the Spanish CICYT (grant MAT94-0058) and DGICYT (grant PB931125). EC is grateful to the "Fundación Cultural Privada Esteban Romero" for partial financial support. 
${ }^{1}$ K. Nakamura, Quantum Chaos (Cambridge University Press, Cambridge 1994).

${ }^{2}$ A. M. Chang, H. U. Baranger, L. N. Pfeiffer, and K. W. West, Phys. Rev. Lett. 73, 2111 (1994).

${ }^{3}$ E. Cuevas, E. Louis and J. A. Vergés, Phys. Rev. Lett. 77, 1970 (1996).

${ }^{4}$ J. A. Vergés, E. Cuevas, E. Louis and M. Ortuño, unpublished.

${ }^{5}$ M. C. Gutzwiller, Chaos in Classical and Quantum Mechanics (Springer-Verlag, New York, 1990).

6 T. A. Brody, J. Flores, J. B. French, P. A. Mello, A. Pandey, and S. S. M. Wong, Rev. Mod. Phys. 53, 385 (1981).

${ }^{7}$ O. Bohigas, in Chaos and Quantum Physics, Proceedings of the Les Houches Summer School, Session L11, 1989, edited by M.-J. Giannoni, A. Voros and J. Zinn-Justin (Elsevier, New York, 1991).

${ }^{8}$ B. L. Altshuler and B. I. Shklovskii, Zh. Eksp. Teor. Fiz. 91, 220 (1986) [Sov. Phys. —JETP 64, 127 (1986)].

${ }^{9}$ A. Altland and Y. Gefen, Phys. Rev. B 51, 10671 (1995).

${ }^{10}$ E. Cuevas, E. Louis, M. Ortuño and J. A. Vergés, unpublished.

${ }^{11}$ H. R. Schwarz, Numer. Math. 12, 231 (1968).

12 E. N. Economou, Green's Functions in Quantum Physics (Springer-Verlag, Berlin, 1983).

13 P. Sheng, Introduction to Wave Scattering, Localization, and Mesoscopic Systems (Academic Press, San Diego, 1995).

${ }^{14}$ Regions of small density of states favor the appearance of slightly perturbed states because larger energy separations imply a diminution of mixing among the states.

${ }^{15}$ G. Casati, B. V. Chirikov, and I. Guarneri, Phys. Rev. Lett. 54, 1350 (1985).

16 M. L. Mehta, Random Matrices (Academic Press, San Diego 1991).

${ }^{17}$ V. Gasparian, E. Louis and J. A. Vergés, Phys. Rev. B 53, 6967 (1996)

${ }^{18}$ See the work by A. Altland, Y. Gefen and G. Montambaux, Phys. Rev. Lett. 76, 1130 (1996) to see how the standard definition of a transport critical energy done by D. J. Thouless, Phys. Rev. Lett. 39, 1167 (1977) applies to level correlations.

${ }^{19}$ E. Cuevas, E. Louis, M. Ortuño and J. A. Vergés, unpublished.

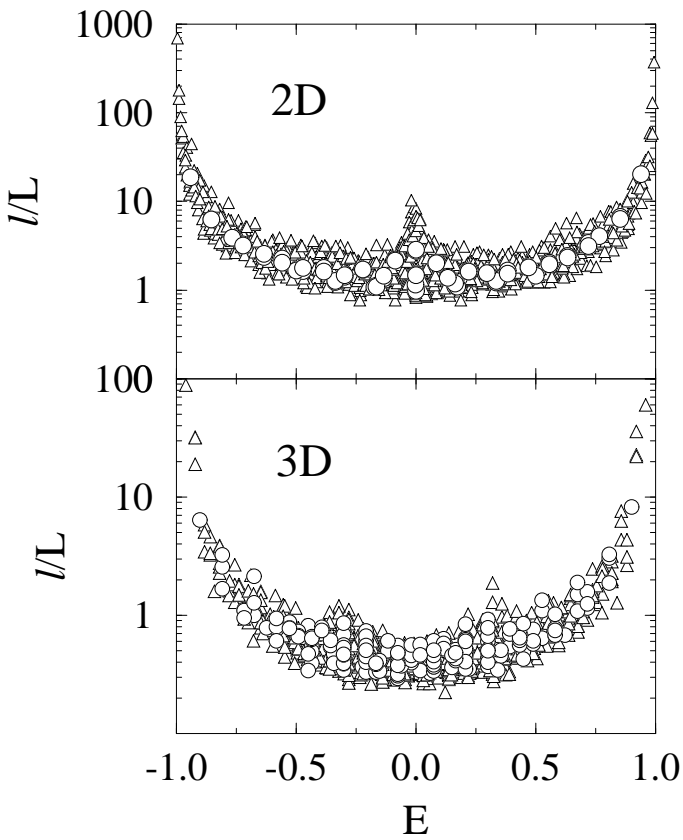

FIG. 1. Mean free path (divided by the system size $L$ ) as a function of the energy (scaled by half the band width, 4 and 6 in 2D and 3D, respectively) for the chaotic billiards discussed in this work. In the $2 \mathrm{D}$ case the results correspond to $L=8$ and 32 (circles and triangles) and $W=2$, and in the $3 \mathrm{D}$ case to $L=6$ and 10 (circles and triangles) and $W=4$. The fact that the data for the two sizes collapse indicates that $l \propto L$ (see text).

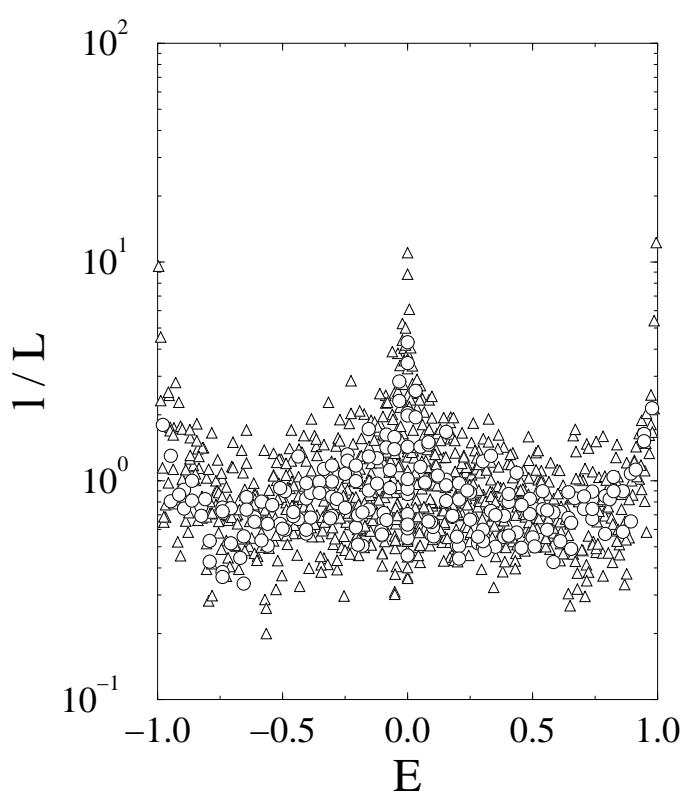


FIG. 2. Mean free path (divided by the system size $L$ ) as a function of the energy (scaled by half the band width) for the chaotic billiards discussed in this work. Results correspond to $L=16$ and 32 (circles and triangles) and $W=10$. The fact that the data for the two sizes collapse indicates that $l \propto L$ (see text).

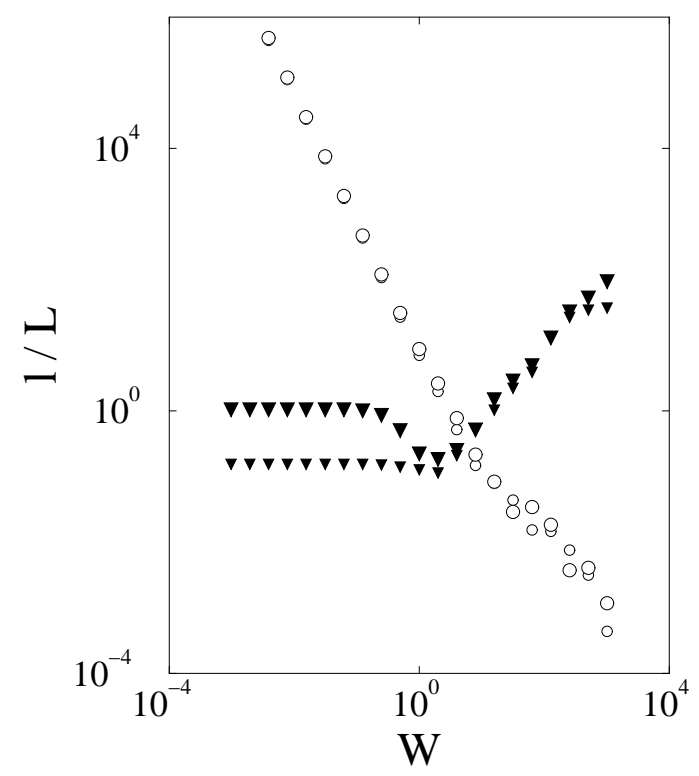

FIG. 3. Mean free path $l$ divided by $L$ as a function of $W$ for the quantum chaotic billiards investigated in this work. Standard approach results are given by circles whereas our alternative calculation is represented by down triangles. Small symbols correspond to $L=16$ and large symbols to $L=32$. The results correspond to an average over an energy window of 0.2 around an energy equal to -2 .

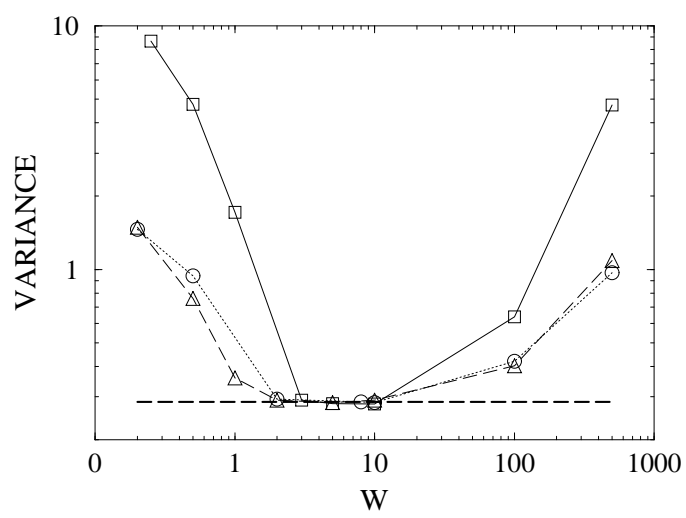

FIG. 4. Variance of the nearest-level spacing distributions as a function of the disorder parameter $W$ for the two (circles, $L=32$, triangles, $L=90$ ) and three (squares, $L=10$ ) dimensional billiards here investigated. The results correspond to an average over an energy window of 0.2 around the energy -1 . The dashed horizontal line corresponds to the GOE variance (0.286).

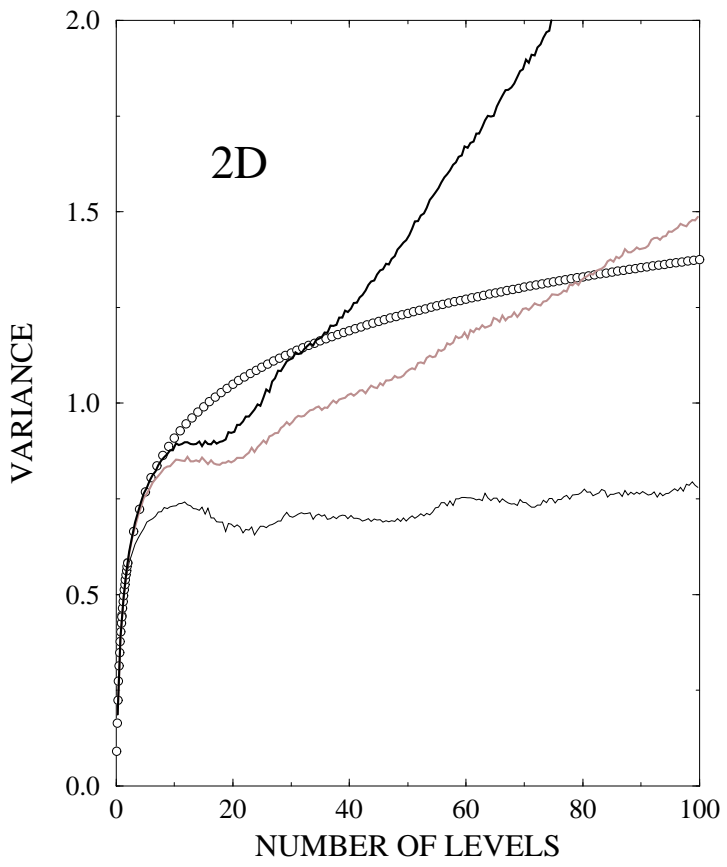

FIG. 5. $\quad \Sigma^{2}(E)$ as a function of $E$ for the 2 D billiard. The results correspond to $L=32$ and disorder $W=2$ (black thin line), $W=6$ (gray thick line), and $W=10$ (black thick line). One thousand samples and all states in the region $-3 \leq \epsilon \leq 3$ are included in the calculation. Circles show the variance value corresponding to GOE fluctuations. 


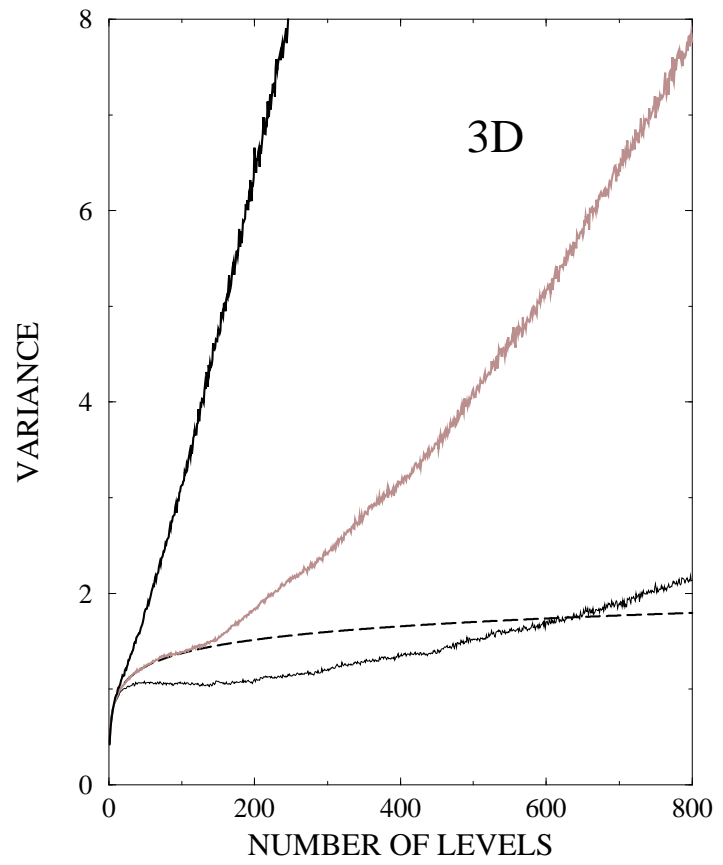

FIG. 6. $\quad \Sigma^{2}(E)$ as a function of $E$ for the 3D billiard. The results correspond to $L=20$ and disorder $W=2$ (black thin line), $W=4$ (gray thick line), and $W=10$ (black thick line). One hundred samples and all states in the region $-4 \leq \epsilon \leq-2$ are included in the calculation. The dashed line shows the variance value corresponding to GOE fluctuations.

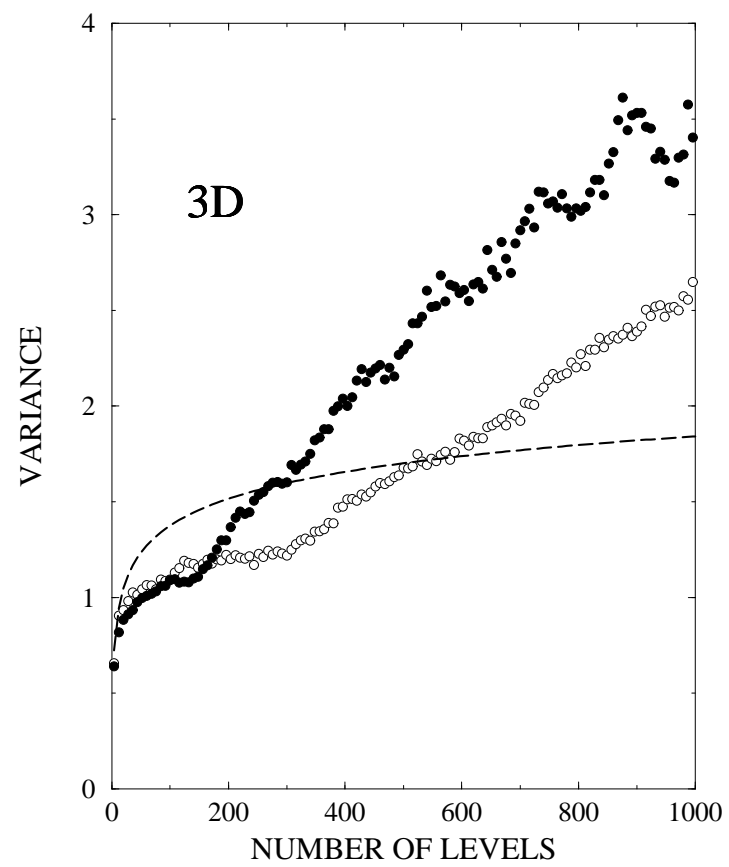

FIG. 7. Number variance as a function of the mean number of levels of a 3D billiard of surface roughness given by $W=2$ and two different sizes. Results for $L=14$ are given by filled circles whereas results for $L=20$ are given by empty circles. The corresponding GOE values are shown by the dashed line. 